\title{
Efficacy and safety of immunosuppressive treatment in IgA nephropathy: a meta- analysis of randomized controlled trials
}

\author{
Zheng Zhang ${ }^{1}$, Yue Yang ${ }^{1}$, Shi-min Jiang ${ }^{1,2}$ and Wen-ge Li ${ }^{1 *}$
}

\begin{abstract}
Background: Immunosuppressive agents have been widely used in the treatment of IgA nephropathy (IgAN), but the efficacy and safety remain controversial. The recent STOP-IgAN and TESTING studies have again focused attention on the application of immunosuppressive agents in IgAN. This study investigated the benefits and risks of immunosuppressive agents in IgAN.

Methods: MEDLINE, EMBASE, the Cochrane Library, and article reference lists were searched for randomized controlled trials (RCTs) comparing immunosuppressive agents with any other non-immunosuppressive agents for treating IgAN. A meta-analysis was performed on the outcomes of proteinuria, creatinine $(\mathrm{Cr})$, estimated glomerular filtration rate (eGFR), and adverse events in patients with IgAN, and trial sequential analyses were also performed for outcomes.

Results: Twenty-nine RCTs (1957 patients) that met our inclusion criteria were identified. Steroids (weighted mean difference [WMD] -0.70, 95\% confidence interval [CI] -1.2 to - 0.20), non-steroidal immunosuppressive agents (NSI) (WMD $-0.43,95 \% \mathrm{Cl}-0.55$ to -0.31 ), and combined steroidal and non-steroidal immunosuppressive agents (S\&NSI) (WMD $-1.46,95 \% \mathrm{Cl}-2.13$ to -0.79 ) therapy significantly reduced proteinuria levels compared with the the control group. Steroid treatment significantly reduced the risk of end-stage renal disease (ESRD) (relative risk [RR] 0.39, Cl 0.19 to 0.79) compared with the control group. The immunosuppressive therapy group showed significant increases in gastrointestinal, hematological, dermatological, and genitourinary side effects, as well as impaired glucose tolerance or diabetes. Hyperkalemia was more common in the control group.

Conclusion: Immunosuppressive therapy can significantly reduce proteinuria and ESRD risk in patients with IgAN, but with a concomitant increase in adverse reactions. Therefore, care is required in the application of immunosuppressive agents in IgAN.
\end{abstract}

Keywords: IgA nephropathy, Immunosuppressive agents

\section{Background}

IgA nephropathy (IgAN) is one of the most common primary glomerular diseases [1]. A systematic review demonstrated an overall population incidence of IgAN of 2.5/ $100000 / y e a r$ [2]. There is still no uniform standard of treatment for IgAN. The treatment of IgAN mainly includes reducing urinary protein and blood pressure (mainly with angiotensin-converting enzyme inhibitors (ACEIs) and angiotensin receptor blockers (ARBs)), glucocorticoid therapy, non-hormone immunosuppressive therapy, and other

\footnotetext{
* Correspondence: wenge_lee2002@126.com

${ }^{1}$ Department of Nephrology, China-Japan Friendship Hospital, Beijing, China Full list of author information is available at the end of the article
}

treatments. The 2012 Kidney Disease: Improving Global Outcomes (KDIGO) guidelines [3] for IgAN recommend treatment with a renin-angiotensin system (RAS) blocker, such as ACEIs and ARBs, in patients with proteinuria with protein excretion $>1 \mathrm{~g} /$ day. Corticosteroid therapy can be considered in patients with proteinuria $>1 \mathrm{~g} /$ day after 3-6 months of best supportive treatment and without renal failure. Intensive immunosuppression is reserved for patients with crescents in more than half the glomeruli and a rapid decline in renal function. In the past, we believed that the application of immunosuppressants could bring more benefits to IgAN patients with middle and high risk.

(c) The Author(s). 2019 Open Access This article is distributed under the terms of the Creative Commons Attribution 4.0 International License (http://creativecommons.org/licenses/by/4.0/), which permits unrestricted use, distribution, and reproduction in any medium, provided you give appropriate credit to the original author(s) and the source, provide a link to the Creative Commons license, and indicate if changes were made. The Creative Commons Public Domain Dedication waiver (http://creativecommons.org/publicdomain/zero/1.0/) applies to the data made available in this article, unless otherwise stated. 
The publication of the Supportive versus Immunosuppressive Therapy of Progressive IgA Nephropathy (STOP-IgAN) trial in 2015 and Therapeutic Evaluation of Steroids in IgA Nephropathy Global (TESTING) trial in 2017 focused attention on the treatment of IgAN with immunosuppressive agents. According to the results of these two large randomized controlled trials (RCTs), there is still no clear evidence that immunosuppressive therapy can improve the prognosis of IgAN. Therefore, we retrieved RCTs on immunosuppressive therapy for IgAN, and performed a meta-analysis of the efficacy and safety of immunosuppressive therapy in this disease.

Immunosuppressive agents were divided into three subgroups for this meta-analysis: steroids, non-steroidal immunosuppressive (NSI) agents (NSI agents can be seen as steroid-sparing but not as steroid replacing agents), and steroids combined with non-steroidal immunosuppressive (S\&NSI) agents. Their efficacy and safety were compared relative to controls for the treatment of IgAN.

This meta-analysis was performed in accordance with the recommendations of the Cochrane handbook for systematic reviews of interventions [4] and is reported in compliance with the Preferred Reporting Items for Systematic Reviews and Meta-Analyses (PRISMA) statement guidelines [5].

\section{Methods}

\section{Inclusion and exclusion criteria}

This investigation required studies to meet the following inclusion criteria: the study was an RCT; the study compared different immunosuppressive agents versus nonimmunosuppressive agents/placebo/no treatment; and study subjects were adult or pediatric patients with biopsy-proven IgAN.

Studies were rejected according to the following exclusion criteria: immunosuppressant not given orally or intravenously (intestinal steroid budesonide was excluded though 20\% bioavailable); study subjects with secondary IgAN; no data available for this study in the article, data included in other articles, or data repeated in other articles; and article not in English.

\section{Data sources and searches}

The MEDLINE, EMBASE, and Cochrane Library medical databases were searched to retrieve relevant studies. Searches were performed in English, and each search retrieved studies that were published between establishment of the database and May 2018.

A comprehensive search strategy was established to ensure the comprehensive and accurate retrieval of studies. The MEDLINE and Cochrane Library databases were searched using the method described in the Cochrane Policy Manual [6], whereas EMBASE was searched using a sensitivity-specificity filter optimized [7]. The following search terms were used: IgAN, steroids, glucocorticoids, immunosuppressive agents, angiotensin-converting enzyme inhibitors, angiotensin receptor antagonists, and placebo. Furthermore, we also searched relevant professional journals manually.

\section{Data extraction and quality assessment}

Two investigators ( $\mathrm{ZZ}$ and $\mathrm{YY}$ ) independently selected studies from the retrieved literature and extracted the data and analytical results. If the two investigators had different opinions about the quality of a study, a third investigator (SMJ) examined the controversial literature and discussed it with the two aforementioned investigators. Data were included only if the three authors achieve consensus regarding the data.

If necessary, daily proteinuria was recalculated as g/ day. Values for eGFR were based on the data provided by the authors of the included studies.

We assessed treatment-related changes based on mean values and standard deviations (SDs) changes between the pre-treatment and post-treatment. As the standard error of the mean (SEM) was used in some studies, we calculated the SD using the formula: SEM $\times$ square root of sample size. In addition, $95 \%$ confidence intervals (CIs) were used in some studies; we calculated the SD using the formula: ((upper limit of $95 \% \mathrm{CI}-$ lower limit of $95 \% \mathrm{CI}) /(2 \times 1.96)) \times \sqrt{ }(\mathrm{n})$. Assessment of the risks of publication bias followed the Cochrane handbook.

\section{Risk of bias assessment}

Two authors (ZZ and YY) independently evaluated risk of bias using the Cochrane risk-of-bias tool [8]. We reviewed each trial and gave a score of bias according to the following criteria: random sequence generation, allocation concealment, blinding of participants and personnel, blinding of outcome assessment, incomplete outcome data, selective reporting, and other bias.

\section{Statistical analyses}

To compare the effects of immunosuppressive agents and control treatment on proteinuria excretion and serum levels of creatinine, data on eGFR and end-stage renal disease (ESRD) were extracted for meta-analyses. Subgroup analyses were performed for each outcome based on the type of immunosuppressive agent.

For continuous outcomes, the differences in means and the 95\% CI in mean change between baseline and end of treatment value were calculated for individual trials, and the weighted mean difference (WMD) was used as a summary estimator. Dichotomous outcome data from individual trials were analyzed using the relative risk (RR) measure and 95\% CI. Heterogeneity of 
treatment effects between studies was investigated visually by examination of plots and statistically using the heterogeneity $\chi^{2}$ and $\mathrm{I}^{2}$ statistics. In all analyses, $P<0.05$ was taken to indicate statistical significance. The fixedeffects and random-effects models were used for the meta-analysis of each indicator. Analyses were performed using Review Manager 5.2 (RevMan; Cochrane Collaboration, Oxford, UK).

\section{Trial sequential analyses}

To assess whether the current meta-analysis had a adequate sample size to draw firm conclusions about the effects of interventions, we performed trial sequential analyses (TSAs) for outcomes. TSAs involves a cumulative meta-analysis to create a $\mathrm{Z}$ curve of the summarized observed effect and the monitoring boundaries for benefit and harm and estimate the optimal sample size [9]. A sufficient level of evidence for the anticipated intervention effect may have been reached when the cumulative $\mathrm{z}$ curve crosses the trial sequential monitoring boundary. And there is insufficient evidence to reach a conclusion, if the $\mathrm{z}$ curve crosses none of the boundaries and the required information size has not been reached. These analyses were performed using the software TSA version 0.9 Beta (Copenhagen Trial Unit, Copenhagen, Denmark).

\section{Results}

Basic information regarding the included studies

After performing electronic and manual searches, 4016 potentially relevant papers were obtained. After removing duplicated papers, 2639 papers remained. After browsing the titles and abstracts, 53 papers were selected. After reading the entire text of these 53 papers, 24 papers were excluded, and 28 papers describing 25 trials with a total of 1957 patients were ultimately included. The literature selection process is illustrated in Fig. 1, and detailed information regarding the examined studies is provided in Table 1 [10-38].

\section{Quality of trials}

By current standards, reporting of key indicators of trial quality was suboptimal. Some studies in particular provided few details on the process of randomization and concealment of allocation. Only six studies were doubleblinded trials. Seven studies used an open-label design. The bias and overall risk diagrams of the included studies are presented in Fig. 2.

\section{Effects on proteinuria}

The difference in the means of urinary protein excretion between end of treatment and baseline was significantly lower in the steroid group than in controls (five trials

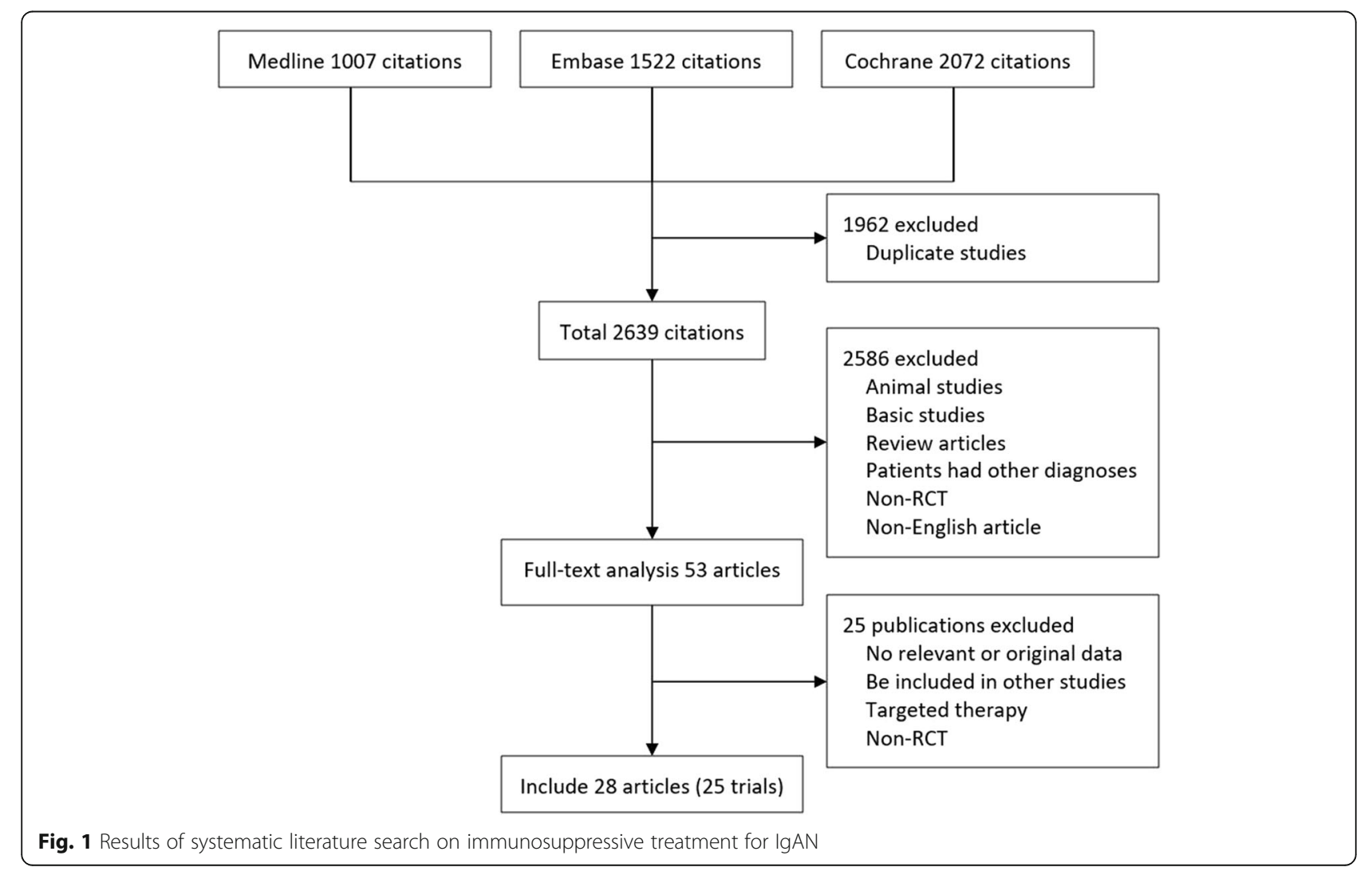


Table 1 Characteristics of RCTs included in the study

\begin{tabular}{|c|c|c|c|c|c|}
\hline Study & Patient & Sample size & Intervention (treatment) & Intervention (control) & Follow-up \\
\hline Ballardie 2002 [10] & 18 to 54 years & $38(19 / 19)$ & $\begin{array}{l}\text { Prednisolone } 40 \mathrm{mg} / \mathrm{d} \\
\text { (reduced to } 10 \mathrm{mg} / \mathrm{d} \text { by } \\
2 \text { year) }+ \text { cyclophosphamide } \\
1.5 \mathrm{mg} / \mathrm{kg} / \text { day (adjusted } \\
\text { down to the nearest } 50 \mathrm{mg} \text { ) }\end{array}$ & no immunosuppression & $24 \mathrm{M}$ \\
\hline Cheng 2015 [11] & $\begin{array}{l}18-55 \text { years old, hypertension } \\
\text { under control, urinary proteins } \\
0.5-3.5 \mathrm{~g} / 24 \mathrm{~h}, \mathrm{Cr}<265.2 \mu \mathrm{mol} / \mathrm{L}\end{array}$ & $84(42 / 42)$ & $\begin{array}{l}\text { leflunomide } 20 \text { mg/d + } \\
\text { Valsartan }\end{array}$ & Valsartan & $24 \mathrm{M}$ \\
\hline Cruzado 2011 [12] & $\begin{array}{l}\text { 18-70 years old, eGFR 30-60 } \\
\mathrm{ml} / \mathrm{min} / 1.73 \mathrm{~m}^{2} \text {, proteinuria } \\
>1 \mathrm{~g} / \mathrm{d} ; \mathrm{BP}>140 / 90 \mathrm{mmHg} \\
\text { with proteinuria } 0.3-1 \mathrm{~g} / \mathrm{d}\end{array}$ & $23(14 / 9)$ & $\begin{array}{l}\text { SRL } 1 \mathrm{mg} / \mathrm{d} \text { (initial) + } \\
\text { enalapril (or ACEI) + } \\
\text { atorvastatin (or } \\
\text { other statin) }\end{array}$ & $\begin{array}{l}\text { Enalapril (or ACEI) + } \\
\text { atorvastatin } \\
\text { (or other statin) }\end{array}$ & $12 \mathrm{M}$ \\
\hline Frisch 2005 [13] & $18-75$ years old, protein $>1 \mathrm{~g} / \mathrm{d}$ & $32(17 / 15)$ & $\begin{array}{l}\text { MMF } 1000 \mathrm{mg} \text { bid } \\
\text { +ACEI/ARB }\end{array}$ & Placebo + ACEI/ARB & $12 \mathrm{M}$ \\
\hline Harmankaya 2002 [14] & $\begin{array}{l}\text { 13-63 years, mean } \mathrm{Ccr} 89.2 \pm \\
10.2 \mathrm{ml} / \mathrm{min}\end{array}$ & $43(21 / 22)$ & $\begin{array}{l}\text { Prednisolone } 40 \mathrm{mg} / \text { day } \\
\text { + azathioprine } 100 \mathrm{mg} / \text { day }\end{array}$ & no specific treatment & $60 M$ \\
\hline Hirai 2017 [15] & $\begin{array}{l}\text { urinary protein excretion } \\
>0.5 \mathrm{~g} / \text { day, age }>16 \text { years }\end{array}$ & $42(21 / 21)$ & $\begin{array}{l}\text { MZR } 150 \text { mg once daily } \\
\text { orally in the morning for } \\
12 \text { months }+ \text { Standard } \\
\text { treatment }\end{array}$ & Standard treatment & $36 \mathrm{M}$ \\
\hline Hogg 2015 [16] & $\begin{array}{l}\text { 7-70 years old; UPCR }>0.6 \mathrm{~g} / \mathrm{g} \\
\text { (males) or }>0.8 \mathrm{~g} / \mathrm{g} \text { (females); } \\
\text { eGFR }>50 \mathrm{~mL} / \mathrm{min} / 1.73 \mathrm{~m} 2 \\
\text { (or }>40 \mathrm{~mL} / \mathrm{min} / 1.73 \mathrm{~m} 2 \text { in } \\
\text { those already receiving ACE } \\
\text { or ARB). }\end{array}$ & $52(25 / 27)$ & $\begin{array}{l}\text { MMF } 25 \text { to } 36 \mathrm{mg} / \mathrm{kg} / \mathrm{d} \\
\text { (Max dose of } 1 \mathrm{~g} / \mathrm{d} \text { ) }+ \\
\text { lisinopril }\end{array}$ & $\begin{array}{l}\text { lisinopril or placebo } \\
25 \text { to } 36 \mathrm{mg} / \mathrm{kg} / \mathrm{d} \\
\text { (Max dose of } 1 \mathrm{~g} / \mathrm{d} \text { ) }\end{array}$ & $12 \mathrm{M}$ \\
\hline Julian 1993 [17] & $\mathrm{Ccr}>25 \mathrm{ml} / \mathrm{min} / 1.73 \mathrm{~m}^{2}$ & $35(17 / 18)$ & prednisone & no placebo & $12 \mathrm{M}$ \\
\hline Yoshikawa 1999a [18] & $<15$ Years old & $78(40 / 38)$ & $\begin{array}{l}\text { Prednisolone } 2 \mathrm{mg} / \mathrm{kg} / \mathrm{d} \\
\text { in three divided doses for } \\
\text { a total dose of not more } \\
\text { than } 80 \mathrm{mg} / \mathrm{d} \text { for } 4 \mathrm{w} \text {, } \\
\text { followed by } 2 \mathrm{mg} / \mathrm{kg} / 2 \mathrm{~d} \text {, } \\
\text { given as a single dose in } \\
\text { the morning of every other } \\
\text { day for } 4 \mathrm{w}, 1.5 \mathrm{mg} / \mathrm{kg} / 2 \mathrm{~d} \\
\text { for } 4 \mathrm{w} \text {, and } 1 \mathrm{mg} / \mathrm{kg} / 2 \mathrm{~d} \text { for } \\
21 \mathrm{~m}+\text { azathioprine } 2 \mathrm{mg} / \mathrm{kg} / \mathrm{d} \\
\text { in a single morning dose for } \\
24 \mathrm{~m}+\text { heparin-warfarin + } \\
\text { dipyridamole }\end{array}$ & $\begin{array}{l}\text { heparin-warfarin + } \\
\text { dipyridamole }\end{array}$ & $24 \mathrm{M}$ \\
\hline Katafuchi 2003 [19] & $\begin{array}{l}\leq 60 \text { years old, } \mathrm{Cr} \\
<1.5 \mathrm{mg} / \mathrm{dl}(132.6 \mathrm{umol} / \mathrm{L})\end{array}$ & $90(43 / 47)$ & $\begin{array}{l}\text { prednisolone orally: } 20 \mathrm{mg} / \mathrm{d} \\
\text { for } 1 \text { month, followed by } \\
15 \mathrm{mg} / \mathrm{d} \text { for } 1 \text { month, } \\
10 \mathrm{mg} / \mathrm{d} \text { for } 1 \text { month, } \\
7.5 \mathrm{mg} / \mathrm{d} \text { for } 3 \text { months, } \\
\text { and } 5 \mathrm{mg} / \mathrm{d} \text { for } 18 \mathrm{months}+ \\
\text { dipyridamole } 150-300 \mathrm{mg} / \text { day }\end{array}$ & $\begin{array}{l}\text { Dipyridamole 150-300 } \\
\text { mg/day }\end{array}$ & $60 M$ \\
\hline Kim 2013 [20] & $\begin{array}{l}\text { 18-70 years old, serum } \\
\text { creatinine } \leq 1.5 \mathrm{mg} / \mathrm{dL} \text { or } \\
\text { eGFR } \geq 45 \mathrm{ml} / \mathrm{min} / 1.73 \mathrm{~m}^{2} \text {, } \\
\text { UACR } 0.3-3 \mathrm{~g} / \mathrm{g} \text { creatinine, } \\
\text { BP }<130 / 80 \mathrm{mmHg}\end{array}$ & $40(20 / 20)$ & $\begin{array}{l}\text { Tacrolimus } 0.1 \mathrm{mg} / \mathrm{kg} / \text { day, } \\
8 \text { weeks (maintain trough } \\
\text { levels at } 5-10 \mathrm{ng} / \mathrm{ml} \text { ) } \\
\rightarrow 0.05 \mathrm{mg} / \mathrm{kg} / \text { day, } 16 \text { weeks } \\
\text { (maintain the trough level in } \\
5-10 \mathrm{ng} / \mathrm{ml} \text { ) + RASi }(9 / 20)\end{array}$ & RASi(11/20), placebo & $16 \mathrm{~W}$ \\
\hline Koike 2008 [21] & NA & $48(24 / 24)$ & $\begin{array}{l}\text { initially treated with } \\
0.4 \mathrm{mg} / \mathrm{kg} / \text { day of } \\
\text { prednisolone ( } 20-30 \mathrm{mg} / \text { day) } \\
\text { for the first } 4 \text { weeks, and the } \\
\text { dose was gradually reduced } \\
\text { to } 10-20 \mathrm{mg} \text { on alternate } \\
\text { days for the next } 12 \text { months, } \\
\text { and then } 5-10 \mathrm{mg} \text { on alternate } \\
\text { days for a subsequent year }\end{array}$ & $\begin{array}{l}\text { Dipyridamole or dilazep } \\
\text { hydrochloride }\end{array}$ & $24 \mathrm{M}$ \\
\hline
\end{tabular}


Table 1 Characteristics of RCTs included in the study (Continued)

\begin{tabular}{|c|c|c|c|c|c|}
\hline Study & Patient & Sample size & Intervention (treatment) & Intervention (control) & Follow-up \\
\hline Pozzi 1999 [22] & $\begin{array}{l}15-69 \text { years old, urinary } \\
\text { protein excretion of } \\
1.0-3.5 \mathrm{~g} / \mathrm{d}, \mathrm{Cr} \leq 133 \\
\text { umol/L ( } 1.5 \mathrm{mg} / \mathrm{dL})\end{array}$ & $86(43 / 43)$ & $\begin{array}{l}\text { methylprednisolone } \\
\text { intravenously for } 3 \text { consecutive } \\
\text { days; this course was repeated } \\
2 \text { months and } 4 \text { months later. } \\
\text { Oral prednisone was given at } \\
\text { a dose of } 0.5 \mathrm{mg} / \mathrm{kg} \text { on } \\
\text { alternate days for } 6 \text { months. }\end{array}$ & Supportive treatment & $60 M$ \\
\hline Lai 1986 [23] & $\begin{array}{l}14-42 \text { years old, } \\
\text { IgAN \& NS }\end{array}$ & $34(17 / 17)$ & $\begin{array}{l}\text { prednisone/prednisolone } \\
40-60 \mathrm{mg} / \mathrm{d} \text {, reduce by half } \\
\text { after } 8 \text { weeks }\end{array}$ & Supportive therapy & $38 \mathrm{M}$ \\
\hline Lv 2009 [24] & $\begin{array}{l}18-65 \text { years old, urinary } \\
\text { proteins } 1-5 \mathrm{~g} / \mathrm{d} \text {, eGFR> } \\
30 \mathrm{ml} / \mathrm{min}\end{array}$ & $63(33 / 30)$ & $\begin{array}{l}\text { prednisone: } 0.8-1.0 \mathrm{mg} / \mathrm{kg} / \text { day } \\
\text { for } 8 \text { weeks, tapered by } \\
5-10 \mathrm{mg} \text { every } 2 \text { weeks } \\
+ \text { cilazapril }\end{array}$ & cilazapril & $48 \mathrm{M}$ \\
\hline Lv 2017 [25] & $\begin{array}{l}\text { proteinuria> } 1 \mathrm{~g} / \mathrm{d}, \text { eGFR: } \\
20-120 \mathrm{ml} / \mathrm{min} / 1.73 \mathrm{~m}^{2}\end{array}$ & $262(136 / 126)$ & $\begin{array}{l}\text { oral methylprednisolone } \\
(0.6-0.8 \mathrm{mg} / \mathrm{kg} / \mathrm{d} \text {; maximum, } \\
48 \mathrm{mg} / \mathrm{d})\end{array}$ & placebo & $60 \mathrm{M}$ \\
\hline Maes 2004 [26] & $\begin{array}{l}>18 \text { years old, inulin clearance } \\
20-70 \mathrm{~mL} / \mathrm{min} / 1.73 \mathrm{~m}^{2}, \text { proteinuria } \\
>1 \mathrm{~g} / \mathrm{day}, \mathrm{BP}>140 / 90 \mathrm{mmHg}\end{array}$ & $34(21 / 13)$ & MMF: $2 \mathrm{~g} / \mathrm{d}+\mathrm{ACEl}$ & $\begin{array}{l}\text { Placebo (identical } \\
\text { lactose-containing } \\
\text { capsules) }\end{array}$ & $36 \mathrm{M}$ \\
\hline Manno 2009 [27] & $\begin{array}{l}16-70 \text { years old, proteinuria> } \\
1 \mathrm{~g} / \mathrm{d} \text {, eGFR } \geq 50 \mathrm{ml} / \mathrm{min} / 1.73 \mathrm{~m}^{2}\end{array}$ & $97(48 / 49)$ & $\begin{array}{l}\text { prednisone: } 1.0 \mathrm{mg} / \mathrm{kg} / \text { day } \\
\text { (Max: } 75 \mathrm{mg} / \text { day) for } 2 \text { months, } \\
\text { tapered by } 0.2 \mathrm{mg} / \mathrm{kg} / \text { day } \\
\text { every month ramipril }\end{array}$ & ramipril & $5 Y$ \\
\hline Rauen 2015 [28] & $\begin{array}{l}\text { proteinuria }>0.75 \mathrm{~g} / \mathrm{d} \text { after } \\
6 \text { months support treatment }\end{array}$ & $162(82 / 80)$ & $\begin{array}{l}\text { Supportive Care }(100 \%)+ \\
\text { Immunosuppression }\end{array}$ & RASi $(77 / 80)$ & $36 \mathrm{M}$ \\
\hline Shoji 2000 [29] & $\begin{array}{l}15-55 \text { years old, proteinuria } \\
\text { less than } 1.5 \mathrm{~g} / \mathrm{d} \text {, serum creatinine } \\
\text { level less than } 1.5 \mathrm{mg} / \mathrm{dL}\end{array}$ & 19(11/8) & $\begin{array}{l}\text { prednisolone } 0.8 \mathrm{mg} / \mathrm{kg} \text { of } \\
\text { body weight; this was } \\
\text { gradually reduced to a } \\
\text { daily dose of } 0.4 \mathrm{mg} / \mathrm{kg} \\
\text { of body weight during } \\
\text { the first month of therapy, } \\
\text { and then tapered to } 10 \mathrm{mg} \\
\text { very other day for the } \\
\text { remainder of the } 1 \text { year } \\
\text { of therapy }\end{array}$ & $\begin{array}{l}\text { Dipyridamole } \\
300 \mathrm{mg} / \text { day }\end{array}$ & $12 \mathrm{M}$ \\
\hline Tang $2005^{\mathrm{c}}[30]$ & $\begin{array}{l}\text { urinary proteins }>1 \mathrm{~g} / \mathrm{d}, \mathrm{BP} \\
<125 / 85 \mathrm{mmHg} \\
\mathrm{Cr}<300 \mathrm{umol} / \mathrm{L}(3.4 \mathrm{mg} / \mathrm{dl})\end{array}$ & $40(20 / 20)$ & $\begin{array}{l}\text { MMF } 2 \mathrm{~g} / \text { day (weight } \geq 60 \mathrm{~kg} \text { ), } \\
1.5 \mathrm{~g} / \text { day }(\text { weight }<60 \mathrm{~kg}) \\
+ \text { ACEI/ARB }(16: 4)\end{array}$ & ACEI/ARB (14:6) & $72 \mathrm{~W}$ \\
\hline Walker 1990 [31] & $\begin{array}{l}24 \mathrm{~h} \text { pro }>1.0 \mathrm{~g} / \mathrm{d}, 120 \mathrm{umol} / \mathrm{L} \\
<\mathrm{Cr}<200 \mathrm{umol} / \mathrm{L} \text { one or more }\end{array}$ & $52(25 / 27)$ & $\begin{array}{l}\text { Cyclophosphamide } \\
\text { ( } 1-2 \mathrm{mg} / \mathrm{kg} / 24 \mathrm{~h} \text { - maximum } \\
\text { of } 100 \mathrm{mg} / 24 \mathrm{~h} \text { and ajusted } \\
\text { according to peripheral white } \\
\text { cell counts) + dipyridamole } \\
\text { +warfarin }\end{array}$ & no treatment & $2 Y$ \\
\hline Wu 2016 [32] & $\begin{array}{l}18-55 \text { years, proteinuria of } \\
0.5-3.5 \mathrm{~g} / \mathrm{d} \text {, serum creatinine } \\
<265 \mu \mathrm{mol} / \mathrm{L} \text {, blood pressure } \\
\text { between } 90 / 60 \text { and } 130 / 80 \mathrm{mmHg}\end{array}$ & $399(100 / 299)$ & $\begin{array}{l}\text { Leflunomide } 20 \mathrm{mg} / \mathrm{d}+ \\
\text { telmisartan }+ \text { clopidogrel } \\
\text { placebo }\end{array}$ & $\begin{array}{l}\text { Telmisartan + Leflunomide } \\
\text { placebo + clopidogrel } \\
\text { placebo \& Telmisartan } \\
\text { + clopidogrel + } \\
\text { Leflunomide placebo \& } \\
\text { Telmisartan + clopidogrel }\end{array}$ & $24 w$ \\
\hline Xie 2011 [33] & $\begin{array}{l}\text { 14-70 years old, urinary protein } \\
\text { excretion: } 0.5 \text { to } 3.5 \mathrm{~g} / 24 \mathrm{~h} \text {, } \\
\mathrm{Cr}<353.6 \mathrm{umol} / \mathrm{L}\end{array}$ & $64(34 / 30)$ & $\begin{array}{l}\text { MZR } 200 \mathrm{mg} / \mathrm{d} \text { (weight < } 50 \mathrm{~kg}) \\
250 \mathrm{mg} / \mathrm{d}(\text { weight }>50 \mathrm{~kg}) \\
150 \mathrm{mg} / \mathrm{d}(\mathrm{Cr}>176.8 \mathrm{umol} / \mathrm{L}) \\
+ \text { losartan }\end{array}$ & Losartan & $12 \mathrm{M}$ \\
\hline Woo 1987 [34] & & $48(27 / 21)$ & $\begin{array}{l}\text { cyclophosphamide } 1.5 \mathrm{mg} / \mathrm{kg} \\
\text { per day+ dipyridamole + warfarin }\end{array}$ & No treatment & $36 \mathrm{M}$ \\
\hline
\end{tabular}




\begin{tabular}{|c|c|c|c|c|c|c|c|}
\hline & 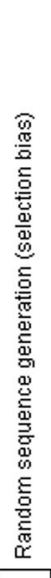 & 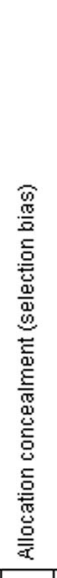 & 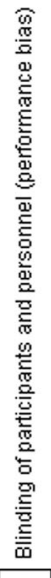 & 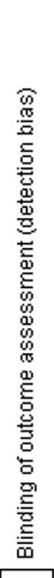 & 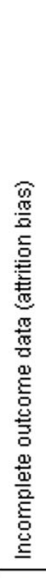 & 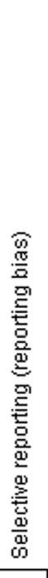 & 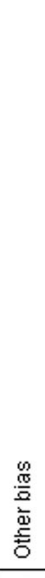 \\
\hline Ballardie 2002 & $?$ & $?$ & $?$ & + & $?$ & $?$ & + \\
\hline Cheng 2015 & + & + & $\odot$ & + & $?$ & $?$ & + \\
\hline Cruzado 2011 & $\odot$ & $?$ & $?$ & + & $?$ & $?$ & + \\
\hline Frisch 2005 & + & + & + & + & $\theta$ & $?$ & + \\
\hline Harmankaya 2002 & $?$ & $?$ & $?$ & + & $?$ & $?$ & + \\
\hline Hirai 2017 & $?$ & $?$ & $?$ & + & $?$ & $?$ & 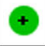 \\
\hline Hogg 2015 & + & + & $\odot$ & + & $\theta$ & $?$ & + \\
\hline Julian 1993 & + & + & $?$ & + & 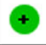 & $?$ & 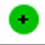 \\
\hline Kamei 2011 & $?$ & $?$ & $?$ & $?$ & $?$ & $?$ & + \\
\hline Katafuchi 2003 & $?$ & $?$ & $?$ & $?$ & $\theta$ & $?$ & $\odot$ \\
\hline Kim 2013 & $\odot$ & + & $\odot$ & + & 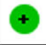 & $?$ & + \\
\hline Koike 2008 & $?$ & $?$ & $\theta$ & $?$ & $?$ & $?$ & + \\
\hline Locatelli 2001 & $?$ & $?$ & $?$ & + & $\odot$ & $?$ & $\odot$ \\
\hline Lv 2009 & + & + & ○ & ? & $\odot$ & $?$ & $\odot$ \\
\hline LV 2017 & $\odot$ & $\odot$ & $\odot$ & + & 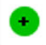 & $?$ & + \\
\hline Maes 2004 & $?$ & $?$ & $?$ & $?$ & $?$ & $?$ & + \\
\hline Manno 2009 & $\odot$ & + & ○ & $?$ & $\theta$ & $?$ & 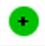 \\
\hline Pozzi 1999 & + & + & $?$ & $?$ & 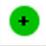 & $?$ & + \\
\hline Pozzi 2004 & $?$ & $?$ & $?$ & $?$ & 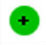 & $?$ & + \\
\hline Rauen 2015 & + & + & $\theta$ & $?$ & $\oplus$ & $?$ & + \\
\hline Shoji 2000 & + & + & $?$ & $?$ & $?$ & $?$ & 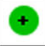 \\
\hline Tang 2005 & $?$ & $?$ & $?$ & $?$ & 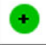 & $?$ & 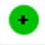 \\
\hline Tang 2010 & + & 0 & $\theta$ & $?$ & + & $?$ & + \\
\hline Walker 1990 & $?$ & $?$ & $?$ & $?$ & $?$ & $?$ & + \\
\hline Woo 1987 & $?$ & $?$ & ○ & $?$ & 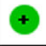 & $?$ & 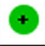 \\
\hline Wu 2016 & $\odot$ & + & $\odot$ & $?$ & $\odot$ & $?$ & $\odot$ \\
\hline Xie 2011 & $?$ & $?$ & $\theta$ & $?$ & + & $?$ & + \\
\hline Yoshikawa 1999 & $\odot$ & + & $?$ & $?$ & 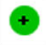 & $?$ & + \\
\hline
\end{tabular}

Fig. 2 Risk of bias graph
[17, 21, 23, 29, 36], 222 patients; WMD -0.51, 95\% CI 0.73 to -0.28 , with a fixed-effects model; WMD -0.70 , $95 \%$ CI -1.2 to -0.20 , with a random-effects model; $\mathrm{I}^{2}=58 \%$; Fig. 3). After removing Lai [23], heterogeneity $\mathrm{I}^{2}$ changed to 0.

Patients receiving NSI alone showed a more significant reduction of urinary protein excretion after treatment compared to controls (seven trials [12, 26, 30-34], 660 patients, WMD $-0.43,95 \% \mathrm{CI}-0.55$ to 0.31 , with a fixed-effects model; WMD $-0.43,95 \% \mathrm{CI}-0.55$ to 0.31 , with a random-effects model; $\mathrm{I}^{2}=0$; Fig. 3 ).

With the S\&NSI treatment approach, patients had a more significant reduction of urinary protein excretion after treatment compared to controls (three trials $[10,18,28], 278$ patients, WMD $-0.16,95 \%$ CI -1.8 to $-1.4, \mathrm{I}^{2}=83 \%$, with a fixed-effects model; WMD $1.42,95 \%$ CI -2.18 to $-0.66, \mathrm{I}^{2}=89 \%$, with a random-effects model; Fig. 3). After removing Yoshikawa [18], heterogeneity $\mathrm{I}^{2}$ changed to 0 .

TSAs of steroids, NSI, and S\&NSI all indicated that the cumulative $\mathrm{z}$ curve crossed both the conventional boundary and the trial sequential monitoring boundary (Fig. 4).

\section{Creatinine}

There were no statistically significant differences in creatinine changes between baseline and end of treatment between immunosuppressive treatment and control groups (nine trials $[11,13,17,19,20,23,26,29,31], 420$ patients, WMD $-0.03,95 \% \mathrm{CI}-0.11$ to 0.15 , with a fixed-effects model; WMD $-0.03,95 \%$ CI -0.11 to 0.05 , with a random-effects model; $\mathrm{I}^{2}=0 \%$; Fig. 5).

TSAs of nine comparisons illustrated that the cumulative $\mathrm{z}$ curve did not cross the conventional boundary or the line of required information size, indicating that the evidence was insufficient. Therefore, further trials are required.

\section{eGFR}

The differences in the means of eGFR between end of treatment and baseline were significantly higher in the NSI group than in controls (five trials [16, 20, 25, 32, 33], 817 patients; WMD 5.17, 95\% CI 3.18 to 7.16 , with a fixed-effects model; WMD 5.17, 95\% CI 3.18 to 7.16 , with a random-effects model; $\mathrm{I}^{2}=0 \%$; Fig. 6). TSAs of five comparisons indicated that the cumulative $\mathrm{z}$ curve crossed the conventional boundary, but did not cross the trial sequential monitoring boundary.

However, when the steroid and S\&NSI groups were added, there were no significant differences in eGFR changes in immunosuppressive treatment compared to controls (seven trials [16, 20, 25, 28, 29, 32, 33], 998 patients, WMD $0.26,95 \% \mathrm{CI}-0.03$ to 0.56 , with a fixed-effects model; WMD $2.52,95 \%$ CI -0.49 to 0.53 , with a 


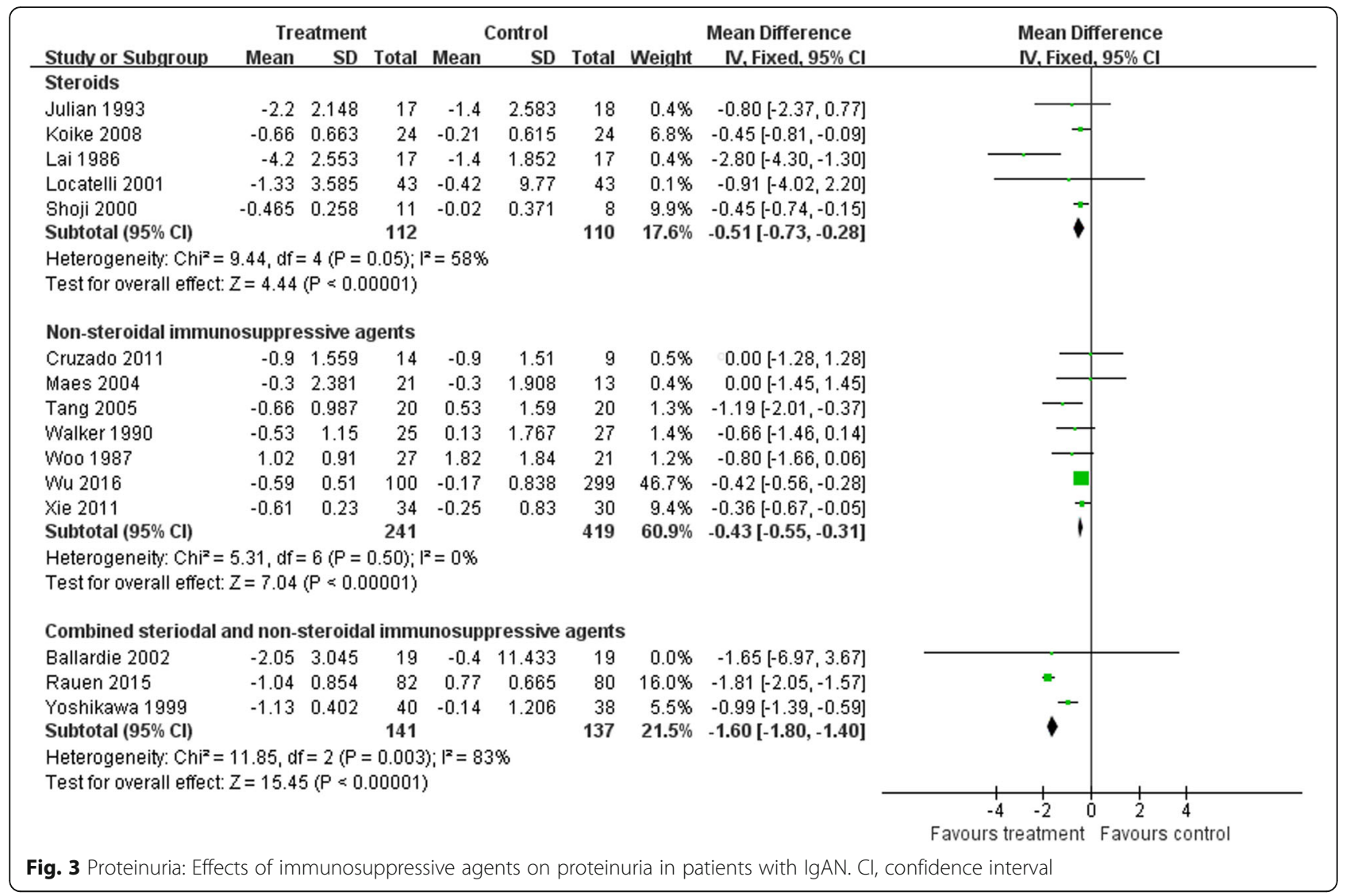

random-effects model; $\mathrm{I}^{2}=76 \%$; Fig. 6). TSAs of seven comparisons indicated that the cumulative $\mathrm{z}$ curve did not cross the conventional boundary or the line of required information size.

\section{ESRD}

There was a lower risk of reaching ESRD in the immunosuppressive treatment group than in controls (12 trials [13, 17, 19, 24-28, 31, 35, 37, 38], 1031 patients; RR $0.51,95 \%$ CI 0.33 to 0.08 , with a fixed-effects model; RR 0.55, 95\% CI 0.33-0.90, with a random-effects model;
$\mathrm{I}^{2}=8$; Fig. 6). These analyses were dominated by the steroid treatment group (Fig. 7).

TSAs of steroids indicated that the cumulative $\mathrm{z}$ curve crossed both the conventional boundary and the trial sequential monitoring boundary.

\section{Adverse events of treatment}

A total of 20 articles reported adverse events during the observation period. The types of adverse events varied widely, and included infection, cardiovascular disease, respiratory disease, hepatotoxicity, and many others; the 13 most commonly reported are listed in
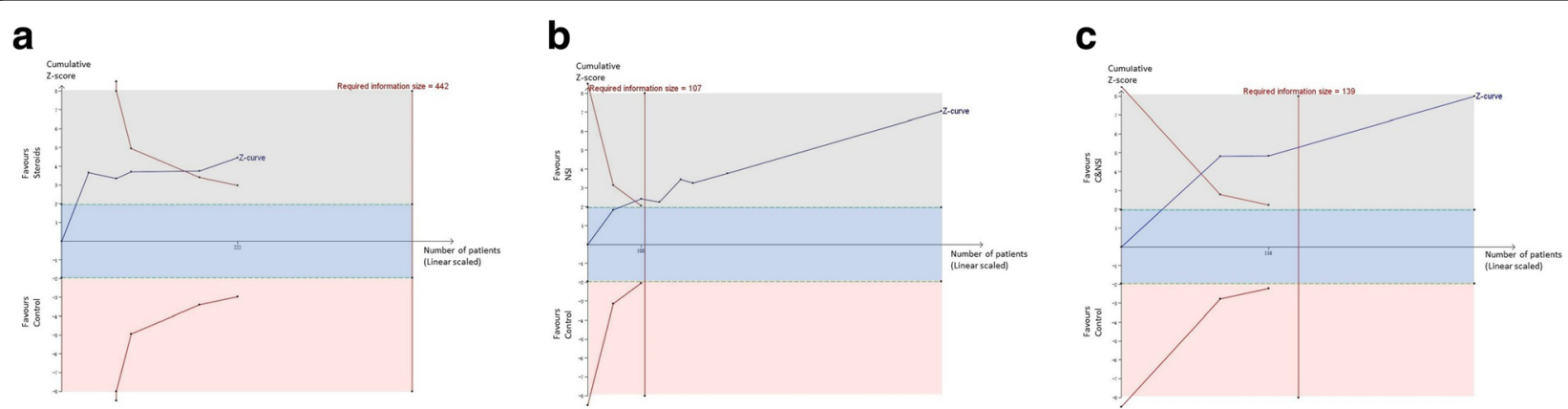

Fig. 4 Trial sequential analyses of proteinuria. a Five comparisons between steroids and controls. b Seven comparisons between NSI and controls c Three comparisons between S\&NSI and controls. Effects on renal function and renal survival 


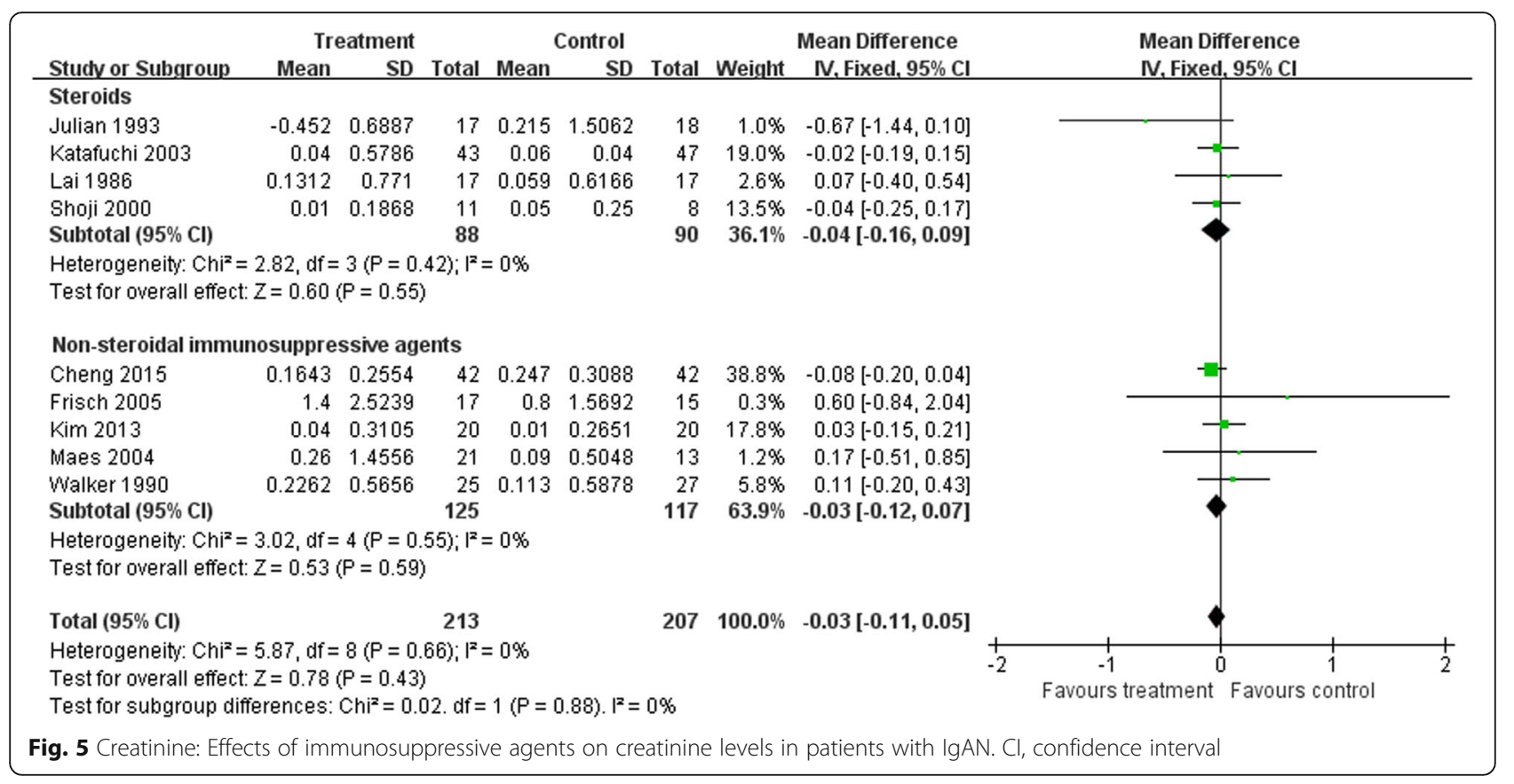

Table 2. As the number of infections reported in Rauen [28] was greater than the total number, RR could not be calculated for infections. TSAs of infection, gastrointestinal disease, hematological disease, dermatological disease, impaired glucose tolerance or diabetes mellitus, and hyperkalemia indicated that the cumulative $\mathrm{z}$ curve crossed the conventional boundary but did not cross the trial sequential monitoring boundary. In addition, TSAs of the other seven diseases indicated that the cumulative $\mathrm{z}$ curve did not cross the conventional boundary or the line of required information size.

\begin{tabular}{|c|c|c|c|c|c|c|c|c|c|}
\hline \multirow[b]{2}{*}{ Study or Subgroup } & \multicolumn{2}{|r|}{ Treatment } & \multicolumn{3}{|c|}{ Control } & \multirow[b]{2}{*}{ Total } & \multirow[b]{2}{*}{ Weight } & \multirow{2}{*}{$\begin{array}{l}\text { Mean Difference } \\
\text { IV. Fixed, 95\% Cl }\end{array}$} & \multirow{2}{*}{$\begin{array}{l}\text { Mean Difference } \\
\text { IV, Fixed, } 95 \% \mathrm{Cl}\end{array}$} \\
\hline & Mean & SD & Total & Mean & SD & & & & \\
\hline \multicolumn{10}{|l|}{ Steroids } \\
\hline Shoji 2000 & 0.102 & 0.28508 & 11 & -0.04864 & 0.3578 & 8 & $97.3 \%$ & $0.15[-0.15,0.45]$ & \\
\hline Subtotal $(95 \% \mathrm{Cl})$ & & & 11 & & & 8 & $97.3 \%$ & $0.15[-0.15,0.45]$ & \\
\hline \multicolumn{10}{|c|}{ Heterogeneity: Not applicable } \\
\hline \multicolumn{10}{|c|}{ Test for overall effect: $Z=0.98(P=0.32)$} \\
\hline \multicolumn{10}{|c|}{ Non-steroidal immunosuppressive agents } \\
\hline Hogg 2015 & -8.6 & 21.63 & 25 & -10.6 & 79.53 & 27 & $0.0 \%$ & $2.00[-29.17,33.17]$ & \\
\hline Kim 2013 & -2.2 & 22.2785 & 20 & -1.5 & 23.663 & 20 & $0.0 \%$ & $-0.70[-14.94,13.54]$ & \\
\hline LV 2017 & -1.79 & 17.55 & 136 & -6.95 & 21.39 & 126 & $0.4 \%$ & $5.16[0.40,9.92]$ & \\
\hline Wu 2016 & 1.9 & 8.2398 & 100 & -3.47 & 13.851 & 299 & $1.7 \%$ & $5.37[3.12,7.62]$ & - \\
\hline Xie 2011 & -0.64 & 29.2579 & 34 & -4.28 & 30.673 & 30 & $0.0 \%$ & $3.64[-11.10,18.38]$ & \\
\hline Subtotal $(95 \% \mathrm{Cl})$ & & & 315 & & & 502 & $2.2 \%$ & $5.17[3.18,7.16]$ & \\
\hline \multirow{2}{*}{\multicolumn{10}{|c|}{$\begin{array}{l}\text { Heterogeneity: } \mathrm{Chi}^{2}=0.76, \mathrm{df}=4(P=0.94) ; \mathrm{I}^{2}=0 \% \\
\text { Test for overall effect: } Z=5.08(P<0.00001)\end{array}$}} \\
\hline & & & & & & & & & \\
\hline \multicolumn{10}{|c|}{ Combined steroidal and non-steroidal immunosuppressive agents } \\
\hline Rauen 2015 & -4.2 & 14.1 & 82 & -4.7 & 12.3 & 80 & $0.5 \%$ & $0.50[-3.57,4.57]$ & \\
\hline Subtotal $(95 \% \mathrm{Cl})$ & & & 82 & & & 80 & $0.5 \%$ & $0.50[-3.57,4.57]$ & \\
\hline \multirow{2}{*}{\multicolumn{10}{|c|}{$\begin{array}{l}\text { Heterogeneity: Not applicable } \\
\text { Test for overall effect: } Z=0.24(P=0.81)\end{array}$}} \\
\hline & & & & & & & & & \\
\hline & & & 408 & & & & $100.0 \%$ & $0.26[-0.03,0.56]$ & \\
\hline Heterogeneity: Chi $^{2}=$ & $24.60, d$ & $f=6(P=0$ & $0.0004)$ & $1^{2}=76 \%$ & & & & & $\begin{array}{ccccc}1 & 1 & & 1 & 1 \\
-20 & -10 & 0 & 10 & 20\end{array}$ \\
\hline $\begin{array}{l}\text { Test for overall effect } \\
\text { Test for subgroup di }\end{array}$ & $\begin{array}{l}Z=1.74 \\
\text { erences }\end{array}$ & $\begin{array}{l}4(P=0.08) \\
\mathrm{Chi}^{2}=23\end{array}$ & 84. $d f=$ & $=2(P<0.0$ & $0001) 1^{2}$ & $=91.6^{\circ}$ & & & Favours treatment Favours control \\
\hline g. 6 eGFR: Effects C & $\mathrm{nmu}$ & nosuppre & ve a & gents on & estimat & glo & rular & ation rate in pati & th IgAN. Cl, confidence interval \\
\hline
\end{tabular}




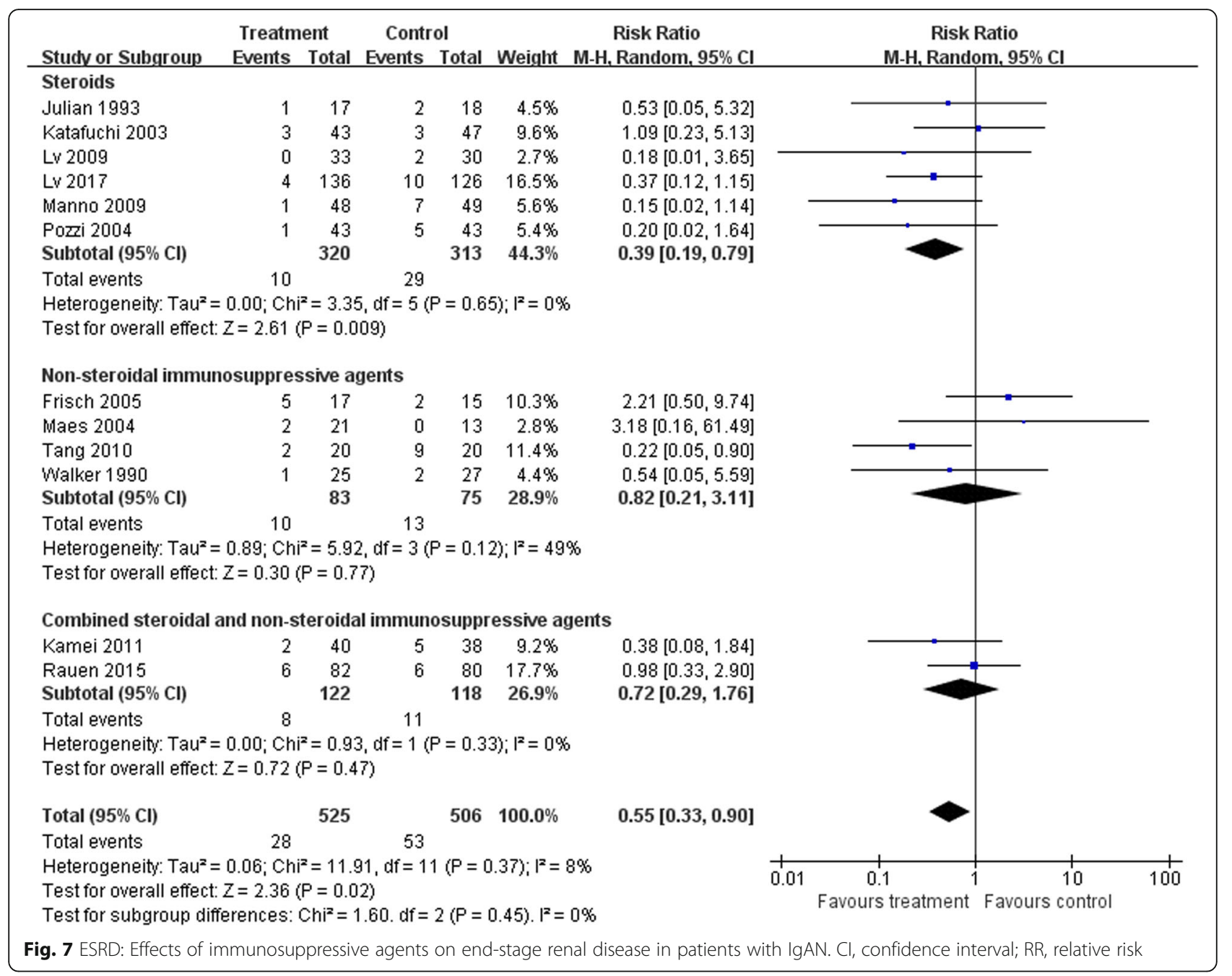

\section{Discussion}

Farnsworth [39] and Barnett [40] first used corticotropin between 1949 and 1950 for the treatment of lipoid nephrosis, which is now known as minimal change disease or childhood nephrotic syndrome. Chasis et al. [41] used nitrogen mustard to treat chronic glomerulonephritis and achieved good initial results, thus pioneering the use of immunosuppressive agents for the treatment of nephropathy. Immunosuppressive agents have been used for the treatment of kidney diseases for about 70 years. However, the outcomes immunosuppressive therapy for IgAN are controversial. Therefore, we included 28 reports published between 1986 and 2017 in a meta-analysis of the efficacy and safety of immunosuppressive treatment and control treatment in IgAN.

\section{Alleviation of proteinuria}

Previous studies have suggested that treatment with steroids or alkylating agents can significantly reduce proteinuria levels in patients with IgAN [42-44]. Our metaanalysis also showed that immunosuppressive agents can significantly reduce the level of proteinuria. The levels of proteinuria in groups treated with steroids, NSI, or S\&NSI were significantly reduced compared to controls. The heterogeneity of the steroid group was mainly derived from Lai [23], in which the inclusion criterion included nephrotic syndrome. In addition, the heterogeneity of the S\&NSI group was mainly derived from Yoshikawa [18], in which the inclusion criterion included age $<15$ years. Sequential analyses showed that immunosuppressive agents were effective for relieving proteinuria, and no additional sample size was required.

\section{Reducing the risk for ESRD}

Our results suggest that non-steroidal immunosuppressive therapy may have a positive effect on eGFR. However, sequential analyses suggested that this is still inconclusive and further studies are required for confirmation. In addition, the treatment group showed a greater reduction in the risk for ESRD than the control group, and this effect was mainly due to the steroid treatment group. Sequential analyses showed that steroids could reduce the risk for 
Table 2 Main adverse events reported in the included RCTs

\begin{tabular}{|c|c|c|c|c|c|c|c|}
\hline \multirow[t]{2}{*}{ Main adverse events } & \multirow{2}{*}{$\begin{array}{l}\text { No. of } \\
\text { studies }\end{array}$} & \multirow{2}{*}{$\begin{array}{l}\text { Immunosuppressive } \\
\text { agent group }\end{array}$} & \multirow{2}{*}{$\begin{array}{l}\text { Control } \\
\text { group }\end{array}$} & \multicolumn{2}{|l|}{$\mathrm{RR}(95 \% \mathrm{Cl})$} & \multicolumn{2}{|l|}{$P$ value } \\
\hline & & & & $\mathrm{FE}$ & RE & $\mathrm{FE}$ & RE \\
\hline Gastrointestinal & 11 & $38 / 431$ & $8 / 606$ & $2.53[1.15,5.55]$ & $2.42[1.07,5.45]$ & 0.02 & 0.03 \\
\hline Hematologic & 9 & $16 / 373$ & $6 / 551$ & $2.17[1.00,4.68]$ & $2.0[0.84,4.77]$ & 0.05 & 0.12 \\
\hline Dermatologic & 7 & $16 / 273$ & $3 / 463$ & $4.09[1.57,10.66]$ & $3.88[1.41,10.64]$ & 0.004 & 0.009 \\
\hline Hepatotoxicity & 7 & $21 / 455$ & $19 / 636$ & $1.26[0.72,2.22]$ & $1.26[0.70,2.24]$ & 0.42 & 0.44 \\
\hline Respiratory & 6 & $9 / 371$ & $12 / 544$ & $0.81[0.37,1.74]$ & $0.82[0.37,1.82]$ & 0.58 & 0.62 \\
\hline Infection & 6 & $189 / 373$ & $114 / 547$ & Not estimable & Not estimable & Not estimable & Not estimable \\
\hline $\begin{array}{l}\text { Impaired glucose tolerance } \\
\text { or diabetes mellitus }\end{array}$ & 5 & $15 / 326$ & $5 / 316$ & $2.61[1.04,6.55]$ & $2.16[0.77,6.05]$ & 0.04 & 0.14 \\
\hline Elevation of blood pressure & 4 & $14 / 193$ & $16 / 389$ & $0.96[0.52,1.79]$ & $0.97[0.43,2.22]$ & 0.9 & 0.95 \\
\hline Malignant & 4 & $4 / 167$ & $2 / 157$ & $1.40[0.39,4.98]$ & $1.33[0.30,5.93]$ & 0.61 & 0.71 \\
\hline Musculoskeletal & 3 & $5 / 238$ & $3 / 226$ & $1.47[0.44,4.93]$ & $1.37[0.40,4.71]$ & 0.53 & 0.62 \\
\hline Hyperkalemia & 3 & $2 / 156$ & $11 / 350$ & $0.23[0.07,0.71]$ & $0.3[0.05,1.98]$ & 0.01 & 0.21 \\
\hline Genitourinary & 3 & $6 / 59$ & $0 / 56$ & $4.59[0.85,24.85]$ & $4.07[0.71,23.39]$ & 0.08 & 0.12 \\
\hline Death & 2 & $3 / 218$ & $2 / 206$ & $1.42[0.24,8.44]$ & $1.41[0.23,8.55]$ & 0.70 & 0.71 \\
\hline
\end{tabular}

$R R$ relative risk, $\mathrm{Cl}$ confidence intervals, $F E$ fixed effect model, $R E$ random effect model

ESRD without the need for a larger sample size. A relevant study [43] also suggested that high-dose short-course steroid therapy has a significant protective effect on renal function, while a low-dose long-course of steroids does not. Further studies are required to determine whether NSI or S\&NSI can reduce the risk for ESRD.

\section{More adverse events}

The use of immunosuppressive agents is often accompanied by side effects. The immunosuppressive therapy group showed significant increases in gastrointestinal, hematological, dermatological, and genitourinary side effects, as well as impaired glucose tolerance or diabetes in this meta-analysis. As the number of infection events reported in the STOP study was too high, even exceeding the total number of patients, it was not possible to calculate the RR value. However, across all studies, the proportion of infections reported was still higher in the immunosuppressive therapy group than in controls. In addition, the TESTING study had to be discontinued because of the excessive number of serious adverse events, mostly infections. By contrast, hyperkalemia was more common in the control group, which may have been related to the application of ACEI and ARB. However, it should be noted that sequential analyses indicated that the statistical results of the above adverse events should be verified by further experiments. Although there were more adverse events in the immunosuppressant group, immunosuppressive agents should be used when necessary because they significantly reduce the risk of ESRD (which means fewer dialysis and transplantation).

\section{Strengths and limitations}

Our study had several limitations that should be taken into consideration. The results of bias analyses indicated that nearly half of the studies did not explicitly report the methods used for randomization. In addition, few studies used blinded methodologies. The quality of the reports in the literature is unsatisfactory. In addition, there were some differences in the inclusion criteria between each study, such as age, proteinuria level, and renal function, and these confounding factors led to a high degree of data heterogeneity. Our results show that glucocorticoids therapy has no significant effect on serum creatinine or eGFR in patients with IgA nephropathy. However, because chronic administration of glucocorticoids significant muscle loss and endogenous creatinine production can ocurr, possible errors in estimation of GFR using serum creatinine based formulas [45] may have led to an over optimistic conclusion.

\section{Conclusions}

In conclusion, the importance of a meta-analysis of the use of immunosuppressant in the treatment of IgA nephropathy was noted. The use of immunosuppressants in the treatment of IgA nephropathy has been shown to significantly reduce proteinuria. Sequential analyzes showed that immunosuppressive agents were effective 
for relieving proteinuria, and no additional sample size was required. In addition, immunosuppressants significantly decrease the risk for ESRD. Immunosuppressant therapy of IgAN has significant benefits. But it also increases the risk for serious adverse reactions. Therefore, in the course of using immunosuppressive agents, close observation should be carried out to prevent and control complications. In addition, further well-designed and high-quality RCTs are needed to explore the applicability and optimal methods of immunosuppressant treatment.

\begin{abstract}
Abbreviations
ACEIs: Angiotensin-converting enzyme inhibitors; ARBs: Angiotensin II receptor blockers; Cls: Confidence intervals; ESRD: End-stage renal disease; IgAN: IgA nephropathy; KDIGO: Improving Global Outcomes; NSI: Nonsteroidal immunosuppressive; RAS: Renin-angiotensin system; RCTs: Randomized controlled trials; RR: Relative risk; S\&NSI: Non-steroidal immunosuppressive; SDs: Standard deviations; SEM: Standard error of the mean; TSAs: Trial sequential analyses; WMD: Weighted mean difference
\end{abstract}

\section{Acknowledgements}

Not applicable.

\section{Authors' contributions}

ZZ designed this study and conducted literature retrieval, data extraction, data analysis and article writing. YY participated in literature retrieval, data extraction and data analysis. SMJ participated in data extraction. WGL participated in study design and literature retrieval, and guided the research. All authors read and approved the final manuscript.

\section{Funding}

There was no funding.

\section{Availability of data and materials}

All data generated or analysed during this study are included in this published article.

\section{Ethics approval and consent to participate}

Not applicable.

\section{Consent for publication}

Not applicable.

\section{Competing interests}

The authors declare that they have no competing interests.

\section{Author details}

'Department of Nephrology, China-Japan Friendship Hospital, Beijing, China. ${ }^{2}$ Graduate School of Peking Union Medical College, Beijing, China.

Received: 22 February 2019 Accepted: 9 August 2019

Published online: 27 August 2019

\section{References}

1. Wyatt RJ, Julian BA. IgA nephropathy. N Engl J Med. 2013;368(25):2402-14.

2. McGrogan A, Franssen CF, de Vries CS. The incidence of primary glomerulonephritis worldwide: a systematic review of the literature. Nephrol Dial Transplant. 2011;26(2):414-30.

3. Cattran DC, Feehally J, Cook HT, Liu ZH, Fervenza FC, Mezzano SA, et al. Kidney disease : improving global outcomes (KDIGO) glomerulonephritis work group. KDIGO clinical practice guideline for glomerulonephritis. Am J Kidney Dis. 2012;2(2):139-274.

4. J.P.T. Higgins G. Cochrane Handbook for systematic reviews of interventions, version 5.1.0. 2011. Available from: http://www.cochrane.org/handbook.

5. Moher D, Liberati A, Tetzlaff J, Altman DG. Preferred reporting items for systematic reviews and meta-analyses: the PRISMA statement. BMJ. 2009; 339.
6. Higgins I GS. Cochrane handbook for systematic reviews of interventions version 5.0. 2 [updated September 2009]. The Cochrane Collaboration, 2009. 2010; Available from www.cochrane-handbook.org.

7. Wong SS, Wilczynski NL, Haynes RB. Developing optimal search strategies for detecting clinically sound treatment studies in EMBASE. J Med Libr Assoc. 2006:94(1):41-7.

8. Higgins JP, Altman DG, Gotzsche PC, Juni P, Moher D, Oxman AD, et al. The Cochrane Collaboration's tool for assessing risk of bias in randomised trials. BMJ. 2011;343:d5928.

9. Wetterslev J, Thorlund K, Brok J, Gluud C. Trial sequential analysis may establish when firm evidence is reached in cumulative meta-analysis. J Clin Epidemiol. 2008;61(1):64-75.

10. Ballardie FW, Roberts ISD. Controlled prospective trial of prednisolone and cytotoxics in progressive IgA nephropathy. J Am Soc Nephrol. 2002;13(1): $142-8$.

11. Cheng G, Liu D, Margetts P, Liu L, Zhao Z, Liu Z, et al. Valsartan combined with clopidogrel and/or leflunomide for the treatment of progressive immunoglobulin a nephropathy. Nephrology (Carlton). 2015;20(2):77-84.

12. Cruzado JM, Poveda R, Ibernón M, Díaz M, Fulladosa X, Carrera M, et al. Low-dose sirolimus combined with angiotensin-converting enzyme inhibitor and statin stabilizes renal function and reduces glomerular proliferation in poor prognosis IgA nephropathy. Nephrol Dial Transplant. 2011;26(11):3596-602.

13. Frisch G, Lin J, Rosenstock J, Markowitz G, D'Agati V, Radhakrishnan J, et al. Mycophenolate mofetil (MMF) vs placebo in patients with moderately advanced IgA nephropathy: a double-blind randomized controlled trial. Nephrol Dial Transplant. 2005; 20(10):[2139-2145]. Available from: http:// cochranelibrary-wiley.com/o/cochrane/clcentral/articles/999/CN-00528999/ frame.html.

14. Harmankaya O, Ozturk Y, Basturk T, Obek A, Kilicarslan I. Efficacy of immunosuppressive therapy in IgA nephropathy presenting with isolated hematuria. Int Urol Nephrol. 2002;33(1):167-71.

15. Hirai K, Ookawara S, Kitano T, Miyazawa H, Ito K, Ueda Y, et al. Efficacy and safety of adding mizoribine to standard treatment in patients with immunoglobulin a nephropathy: a randomized controlled trial. Kidney Res Clin Pract. 2017:36(2):159-66.

16. Hogg RJ, Bay RC, Jennette JC, Sibley R, Kumar S, Fervenza FC, et al. Randomized controlled trial of mycophenolate Mofetil in children, adolescents, and adults with IgA nephropathy. Am J Kidney Dis. 2015;66(5): 783-91.

17. Julian BA, Barker C. Alternate-day prednisone therapy in IgA nephropathy. Preliminary analysis of a prospective, randomized, controlled trial. Contrib Nephrol. 1993;104:198-206.

18. Yoshikawa N, Ito H, Sakai T, Takekoshi Y, Honda M, Awazu M, et al. A controlled trial of combined therapy for newly diagnosed severe childhood IgA nephropathy. J Am Soc Nephrol. 1999;10(1):101-9.

19. Katafuchi R, Ikeda K, Mizumasa T, Tanaka H, Ando T, Yanase T, et al. Controlled, prospective trial of steroid treatment in IgA nephropathy: a limitation of low-dose prednisolone therapy. Am J Kidney Dis. 2003;41(5): 972-83.

20. Kim YC, Chin HJ, Koo HS, Kim S. Tacrolimus decreases albuminuria in patients with IgA nephropathy and normal blood pressure: a double-blind randomized controlled trial of efficacy of tacrolimus on IgA nephropathy. PLoS One. 2013;8(8):e71545.

21. Koike M, Takei T, Uchida K, Honda K, Moriyama T, Horita S, et al. Clinical assessment of low-dose steroid therapy for patients with IgA nephropathy: a prospective study in a single center. Clin Exp Nephrol. 2008;12(4):250-5.

22. Pozzi C, Bolasco P, Fogazzi G, Andrulli S, Altieri P, Ponticelli C, et al. Corticosteroids in IgA nephropathy: a randomised controlled trial. Lancet. 1999;353(9156):883-7.

23. Lai KN, Lai FM, Ho CP, Chan KW. Corticosteroid therapy in IgA nephropathy with nephrotic sydrome: a long-term controlled trial. Clin Nephrol. 1986; 26(4):174-80.

24. LV J, Zhang H, Chen Y, Li G, Jiang L, Singh AK, et al. Combination therapy of prednisone and ACE inhibitor versus ACE-inhibitor therapy alone in patients with IgA nephropathy: a randomized controlled trial. Am J Kidney Dis. 2009; 53(1):26-32

25. Lv J, Zhang $\mathrm{H}$, Wong $M G$, Jardine MJ, Hladunewich $M$, Jha $V$, et al. Effect of Oral methylprednisolone on clinical outcomes in patients with IgA nephropathy: the TESTING randomized clinical trial. JAMA. 2017; 318(5):432-42. 
26. Maes BD, Oyen R, Claes K, Evenepoel P, Kuypers D, Vanwalleghem J, et al. Mycophenolate mofetil in IgA nephropathy: results of a 3-year prospective placebo-controlled randomized study. Kidney Int. 2004;65(5):1842-9.

27. Manno C, Torres DD, Rossini M, Pesce F, Schena FP. Randomized controlled clinical trial of corticosteroids plus ACE-inhibitors with long-term follow-up in proteinuric IgA nephropathy. Nephrol Dial Transplant. 2009;24(12):3694701.

28. Rauen $T$, Eitner F, Fitzner $C$, Sommerer $C$, Zeier $M$, Otte B, et al. Intensive supportive care plus immunosuppression in IgA nephropathy. $\mathrm{N}$ Engl J Med. 2015;373(23):2225-36.

29. Shoji T, Nakanishi I, Suzuki A, Hayashi T, Togawa M, Okada N, et al. Early treatment with corticosteroids ameliorates proteinuria, proliferative lesions, and mesangial phenotypic modulation in adult diffuse proliferative lgA nephropathy. Am J Kidney Dis. 2000;35(2):194-201.

30. Tang S, Leung JC, Chan LY, Lui YH, Tang CS, Kan CH, et al. Mycophenolate mofetil alleviates persistent proteinuria in IgA nephropathy. Kidney Int. 2005; 68(2):802-12 Available from: http://cochranelibrary-wiley.com/o/cochrane/ clcentral/articles/164/CN-00523164/frame.html.

31. Walker RG, Yu SH, Owen JE, Kincaid-Smith P. The treatment of mesangial IgA nephropathy with cyclophosphamide, dipyridamole and warfarin: a two-year prospective trial. Clin Nephrol. 1990;34(3):103-7.

32. Wu J, Duan SW, Sun XF, Li WG, Wang YP, Liu WH, et al. Efficacy of Leflunomide, Telmisartan, and Clopidogrel for immunoglobulin a nephropathy: a randomized controlled trial. Chin Med J. 2016;129(16):1894903.

33. Xie $Y$, Huang $S$, Wang L, Miao L, Zhang A, Li Y, et al. Efficacy and safety of mizoribine combined with losartan in the treatment of IgA nephropathy: a multicenter, randomized, controlled study. Am J Med Sci. 2011;341(5):36772 Available from: http://cochranelibrary-wiley.com/o/cochrane/clcentral/ articles/297/CN-00787297/frame.html.

34. Woo KT, Edmondson RP, Yap HK, WU AY, Chiang GS, Lee EJ, et al. Effects of triple therapy on the progression of mesangial proliferative glomerulonephritis. Clin Nephrol. 1987;27(2):56-64.

35. Kamei K, Nakanishi K, Ito S, Saito M, Sako M, Ishikura K, et al. Long-term results of a randomized controlled trial in childhood IgA nephropathy. Clin J Am Soc Nephrol. 2011;6(6):1301-7.

36. Locatelli F, Pozzi C, Del Vecchio L, Bolasco PG, Fogazzi GB, Andrulli S, et al. Role of proteinuria reduction in the progression of IgA nephropathy. Ren Fail. 2001;23(3-4):495-505.

37. Pozzi C, Andrulli S, Del Vecchio L, Melis P, Fogazzi GB, Altieri P, et al. Corticosteroid effectiveness in IgA nephropathy: long-term results of a randomized, controlled trial. J Am Soc Nephrol. 2004;15(1):157-63.

38. Tang SCW, Tang AWC, Wong SSH, Leung JCK, Ho YW, Lai KN. Long-term study of mycophenolate mofetil treatment in IgA nephropathy. Kidney Int 2010;77(6):543-9.

39. Farnsworth EB. Metabolic changes associated with administration of adrenocorticotropin in the nephrotic syndrome. Proc Soc Exp Biol Med. 1950;74(1):60-2

40. Barnett HL, Mc NH, Mc CW, Forman C, Rapoport M, Michie A, et al. The effects of ACTH and cortisone on the nephrotic syndrome. AMA Am J Dis Child. 1950;80(3):519-20

41. Chasis $\mathrm{H}$, Goldring W, Baldwin DS. Effect of febrile plasma, typhoid vaccine and nitrogen mustard on renal manifestations of human glomerulonephritis. Proc Soc Exp Biol Med. 1949;71(4):565-7.

42. Zhou YH, Tang LG, Guo SL, Jin ZC, Wu MJ, Zang JJ, et al. Steroids in the treatment of IgA nephropathy to the improvement of renal survival: a systematic review and meta-analysis. PLoS One. 2011;6(4):e18788.

43. Lv J, Xu D, Perkovic V, Ma X, Johnson DW, Woodward M, et al. Corticosteroid therapy in IgA nephropathy. J Am Soc Nephrol. 2012;23(6): 1108-16.

44. Cheng J, Zhang X, Zhang W, He Q, Tao X, Chen J. Efficacy and safety of glucocorticoids therapy for IgA nephropathy: a meta-analysis of randomized controlled trials. Am J Nephrol. 2009;30(4):315-22.

45. Ponticelli C, Glassock RJ. Prevention of complications from use of conventional immunosuppressants: a critical review. J Nephrol. 2019.

\section{Publisher's Note}

Springer Nature remains neutral with regard to jurisdictional claims in published maps and institutional affiliations.

\section{Ready to submit your research? Choose BMC and benefit from:}

- fast, convenient online submission

- thorough peer review by experienced researchers in your field

- rapid publication on acceptance

- support for research data, including large and complex data types

- gold Open Access which fosters wider collaboration and increased citations

- maximum visibility for your research: over $100 \mathrm{M}$ website views per year

At $\mathrm{BMC}$, research is always in progress.

Learn more biomedcentral.com/submissions 